\title{
INTELLIGENT TRANSPORTATION SYSTEM BASED TRAFFIC CONGESTION MODELLING FOR URBAN CORRIDOR
}

\author{
Sourabh Jain ${ }^{1}$, Sukhvir Singh Jain ${ }^{2}$ and Gaurav V. Jain ${ }^{3}$ \\ ${ }^{1} \mathrm{Ph}$. D. Research Scholar, Centre for Transportation Systems, \\ Indian Institute of Technology Roorkee, Roorkee, India \\ ${ }^{2}$ Professor, Transportation Engineering Group, Department of Civil Engineering, \\ Indian Institute of Technology Roorkee, Roorkee, India \\ ${ }^{3}$ Scientific Engineer -SE, Space Application Centre (SAC), \\ Indian Space Research Organization (ISRO), Ahmadabad, India
}

\begin{abstract}
This study attempts to make use of traffic behaviour on the aggregate level to estimate congestion on urban arterial and sub-arterial roads of a city exhibiting heterogeneous traffic conditions by breaking the route into independent segments and approximating the origin-destination based traffic flow behaviour of the segments. The expected travel time in making a trip is modelled against sectional traffic characteristics (flow and speed) at origin and destination points of road segments, and roadway and segment traffic characteristics such as diversion routes are also tried in accounting for travel time. Predicted travel time is then used along with free flow time to determine the state of congestion on the segments using a congestion index (CI). A development of this kind may help in understanding traffic and congestion behaviour practically using easily accessible inputs, limited only to the nodes, and help in improving road network planning and management.
\end{abstract}

\section{KEYWORDS}

Congestion, Delay, Origin and Destination, Traffic, Travel Time

\section{INTRODUCTION}

Traffic congestion, not limited to but especially prevalent in metropolitan cities, is one of the most conspicuously worsening problems associated with traffic engineering and urban planning, with clear implications on spheres of urban economy, environment and lifestyle. Traffic in cities continues to grow meteorically especially in major cities of developing countries, which are characterized by heavy economic and population growth. This naturally necessitates intense transportation of goods and passengers, increasing demand for personal vehicular ownership that over the last decade has seen exponential growth worldwide. However, the failure of sufficiently rapid infrastructural development required to cater to this burgeoning traffic frequently leads to failures of the urban transportation system, resulting in traffic jams. Since congestion drastically reduces traffic speed, often bringing it down to near zero, it heavily influences daily life activities, both on the personal as well as the corporate level, and causes annoyance as well as pollution, besides economic loss. Quantification of congestion thus becomes essential in checking congestion by examining the short and long term measures to be undertaken in order to provide a sustainable transportation system that necessitates a well-functioning well-integrated urban economy. Capturing congestion not only becomes important in this respect, but rather essential. 
From engineering point of view, eliminating congestion is not viable, though reducing it significantly by making effective use of traffic movement patterns and network management certainly is.

\section{OBJECTIVE}

Measurement of congestion often takes the path of finding focal regions with high perceptibility of traffic condensation. The viability of origin-destination (O-D) based congestion modelling remains by-and-large unanalyzed. Although O-D studies are widely conducted for network analyses, particularly route choice modelling and in urban planning such as construction of urban rapid transit systems, its value as a source of congestion is seldom underscored, and only a few studies featuring O-D analysis have forayed into congestion estimation.

Furthermore, the complexity of traffic systems in several developing countries is exacerbated due to the prevalence of heterogeneous traffic that only furthers the chaotic nature of the study. This makes it important to study heterogeneous traffic systems in detail and suggest measures for simplification of the different studies already established for homogeneous traffic conditions, such as those in the developed countries.

This study aims to understand the relationship between the traffic conditions of the source and the destination in portions ("segments") of an arbitrarily chosen trip on an arterial and sub-arterial road in a major metropolitan city of India characterizing extremely diverse traffic conditions, and analyze the viability of promoting the use of O-D based measures of congestion to estimate the severity of the problem in the route. For this purpose, the basic traffic parameters, such as volume, speed, density and capacity are measured or calculated at different nodes of the study route and tried against the aforementioned indicator of congestion: Congestion Index, and a review for the prepared model and the behavior of the variables used is then prepared.

\section{RESEARCH SURVEY}

Congestion has been variously defined as a physical condition in traffic streams involving reduced speeds, restrained movement, extended delays and paralysis of the traffic network. It might be characterized as the condition of movement stream on a transportation office portrayed by high densities and low speeds, in respect to some picked reference state with low densities and high speeds [4]. It is a state of movement delay (when the stream of activity is hindered beneath sensible paces) on the grounds that the quantity of vehicles attempting to utilize the street surpasses the activity system ability to handle those [16].

The definition of congestion has been conventionally categorized on the basis of four parameters: capacity, speed, delay/travel time and cost incurred due to congestion. None of these criteria individually describes congestion best, both theoretically and experimentally [8]. Capacity based approaches are common and straightforward, but do not appropriately describe the state of congestion on the microscopic level. The volume-by-capacity ratio $(\mathrm{v} / \mathrm{c})$ is a popular preliminary measure that compares the given traffic conditions with the limiting on-capacity conditions, and is used to assess the Level of Service (LOS) of the road, another commonly used parameter that makes use of various variables primarily focusing on the status of efficiency of the road capacity. Speed based measures of congestion are said to be more efficient than others in explaining the degree of congestion [8]. Anjaneyulu and Nagaraj developed a methodology of determining the state of congestion on road segments with the help of statistical measures of speed such as coefficient of variation of speed. Cost based definition of congestion takes into account the more practical measure of financial losses incurred to the urban economy by estimating the opportunity cost of the time lost in congestion, along with other losses such as that of perishable products. 
Estimation of this opportunity cost involves several variables and by overlooking non-economic aspects, this method fails to provide any insight about the physical state of the traffic system [2]. Chakrabartty and Gupta in 2015 estimated the cost of congestion on a route in Kolkata, based on the methodology devised by R.J. Smeed. [5][14].

The fourth approach, based on delay and/or travel time, is flexible and is said to better describe the meaning of congestion as being closely related to personal loss and annoyance because of delay in making a trip. Congestion can be defined as the travel time or delay incurred in excess of that in light or free flow conditions [8]. Delay in theoretical sense is calculated as the difference of the expected travel time for a trip with the unhindered (free flow) time for the same trip. Time based measures provide a stronger basis for more generalised conclusions and indicators like Travel Time Index (TTI) and its derivative Congestion Index (CI) are easy to comprehend [11], while travel time based Buffer Time Index (BTI) and Planning Time Index (PTI) are reliable in congestion prediction [3]. Congestion Index, which is the ratio of delay experienced in making a trip to the time taken for the same in an unhindered flow (free flow) condition, was introduced in 1992 and is regularly used by the traffic and navigation telematics company TomTom in its official global congestion report [15]. Though several travel time/delay based measures of congestion such as Travel Time, Travel Time Index, Travel Rate Index, Delay Rate, Delay Ratio and Buffer Rate Index exist [1], this study makes use of Congestion Index (CI) because of its ease of calculation and intensive nature as a ratio: it is independent of the path length and other variables that remain constant in the cases for the numerator and the denominator. It was defined as [15]: -

$$
\text { Congestion Index }=\frac{\text { Delay }}{\text { Free Flow Time }}
$$

Alternatively,

$$
C I=\frac{\delta}{T_{f}}=\frac{T-T_{f}}{T_{f}}
$$

Where $\delta$ is delay, $\mathrm{T}$ is the observed/expected travel time and $\mathrm{T}_{\mathrm{f}}$ is free flow time, the time taken for the same trip in unhindered or "free flow" conditions such as at midnight or in early morning. The value of CI depicts the percentage extra time taken by a vehicle in traversing a road segment in comparison to the free flow condition. For example, a value of 0.20 means that the delay experienced is $20 \%$ of the free flow time, or in other words, the trip takes $20 \%$ more time than it ought to (in the ideal conditions). The value of free flow speed (FFS) is dependent on roadway and control conditions, such as carriageway width, clearances, visibility and sight distance, roughness and texture, and night-time intersection signalization. Hall and Vyas considered the value of posted speed limit as the nominal FFS, while Schrank and Lomax took FFS as $60 \mathrm{mph}$ for freeways and $35 \mathrm{mph}$ for arterial roads [13]. In a somewhat perceivably inaccurate estimation of FFS, the 2005 Urban Mobility Report that used TTI approximated FFS as the 85th percentile speed in the off-peak period of the speed profile of the daily traffic.Traffic congestion is dependent on an array of static and dynamic variables, categorised broadly as roadway (path length, space available, etc.), demand/volume based and operational factors (speed, delay, etc.), and a balanced combination of these factors must be employed in estimating the degree of congestion [10]. A combination of volume and operational characteristics was used for congestion modelling [9]. The use of origin and destination (O-D) based congestion estimation in theory is limited. Conventionally, O-D matrices are used for trip planning, traffic management and operation studies [11]. Additionally, even though at macroscopic level traffic flow theory is studied in analogy with fluid dynamics [6], the essence of entry and exit traffic parameters in typical traffic streams has not been appreciated. This may be attributed to the lack of apparent prominent relationship of these factors with congestion as opposed to the more basic 
characteristics pertinent to its explicit definition. This study tries to check if a relation between congestion and the node (or O-D) volume and operational characteristics really exists.

\section{METHODOLOGY}

The first step was to identify a suitable route that includes substantial stretches of both arterial and sub-arterial roads and is often wrought with congestion (regular congestion). Subsequently the route was divided into segments separated by major intersections or points of considerable traffic behavioural shift that might give an insight about the concentration of congested regions. For this purpose, eight nodes were chosen, most of them being major rapid transit bus stops or major intersections. The next step was identification of potential factors. Both roadway as well as traffic parameters were considered, and the congestion parameter to be modelled was fixed (Congestion Index, CI). Once the expected data input was rightly identified, data were collected on site using video camera for recording node based traffic parameters and moving car method for measuring the real travel time. A part of data was initially additionally observed manually on site in order to verify the accuracy of the input. The data were pre-processed and sourcedestination and segment variables were calculated. Finally, all variables found were tested for statistical relationships with the dependent variable, CI, in several combinations using $75 \%$ of the observed data. A multiple regression model yielded only the statistically relevant variables. This model was validated for the remaining $25 \%$ observed data with the help of root mean squared error (RMSE). Free flow time was then calculated for each segment and congestion index was found using the predicted values of travel time and free flow time. Lastly, the importance of the variables found relevant in modelling was discoursed.

\section{STUDY ROUTE}

Delhi is a rapidly growing major city of India that, characterized by heterogeneity of traffic composition, is one of the most important centres of transportation of the country [12]. The study route chosen comprised two sections: a long eastern part of the Inner Ring Road, an access controlled divided arterial way, and Sri Aurobindo Marg, a divided sub-arterial that takes diversion from the Ring Road south of AIIMS, a prominent public hospital of India (as per IRC classification). Each portion consists of three segments separated by a total of eight nodes (points). The total length of the study route is about $27.4 \mathrm{~km}$, excluding a $640 \mathrm{~m}$ long stretch between AIIMS North Gate and AIIMS West Gate that was not used for observations.One important parameter considered in the study was the number of intersections/route diversions found in the route, an indicator of the possibilities of traffic going into/coming from the main traffic stream and is thus partly a measure of the complexity of the system. For this purpose, only major intersections and diversion roads were counted for each segment. Table 1 and 2 include the roadway details of the study route: -

Table 1. Observation node details.

\begin{tabular}{|l|l|}
\hline Node ID & Name \\
\hline 1 & Kashmere gate ISBT (Inter State Bus Terminal) \\
\hline 2 & Sarai kale khan bus station \\
\hline 3 & Andrew ganj main intersection \\
\hline 4 & AIIMS north gate \\
\hline 5 & AIIMS west gate \\
\hline 6 & Green park main intersection \\
\hline
\end{tabular}


Civil Engineering and Urban Planning: An International Journal (CiVEJ ) Vol.4, No.2, June 2017

\begin{tabular}{|l|l|}
\hline 7 & IIT gate bus stop \\
\hline 8 & Mehrauli bus terminal \\
\hline
\end{tabular}

Table 2. Segment roadway details.

\begin{tabular}{|l|l|l|l|l|l|}
\hline Seg. ID & $\begin{array}{l}\text { Source } \\
\text { node ID }\end{array}$ & $\begin{array}{l}\text { Dest. node } \\
\text { ID }\end{array}$ & Length $(\mathbf{K m})$ & No. of lanes & $\begin{array}{l}\text { No. of major } \\
\text { intersections }\end{array}$ \\
\hline Arterial road: - & & & & & \\
\hline 1 & 1 & 2 & 12.100 & 6 & 5 \\
\hline 2 & 2 & 3 & 6.080 & 6 & 3 \\
\hline 3 & 3 & 4 & 1.740 & 6 & 1 \\
\hline Sub arterial road: - & & & & & \\
\hline 4 & 5 & 6 & 0.880 & 6 & 0 \\
\hline 5 & 6 & 7 & 1.350 & 4 & 0 \\
\hline 6 & 7 & 8 & 3.430 & 4 & 2 \\
\hline
\end{tabular}

\section{DAta COLlection}

Data collection primarily involved traffic parameter observation on study points ("nodes") such as categorized vehicular traffic volume and spot speed using manual counting and radar gun respectively in count periods of 15 minutes, and travel time using the moving car method. This was done in two phases: Phase I was a four-day manual data collection period, while Phase II involved manual data extraction from video recording over a period of additional twelve days, bringing the total to 16 days.

The traffic data were collected in six motored vehicular categories: standard cars and vans, two wheelers (scooters and motorbikes), three wheelers (auto-rickshaws), LCV (light commercial vehicles), trucks and buses. Following designated slots as shown in table 3 were fixed for data collection: -

Table 3. Observation time slots.

\begin{tabular}{|l|l|l|l|}
\hline Slot ID & Traffic type & Start & End \\
\hline 1. & Morning peak & $8: 00$ AM & 10:00 AM \\
\hline 2. & Morning non-peak & $11: 00 \mathrm{AM}$ & $1: 00 \mathrm{PM}$ \\
\hline 3. & Evening peak & 5:00 PM & 7:00 PM \\
\hline 4. & Evening non-peak & $10: 00 \mathrm{PM}$ & $12: 00 \mathrm{PM}$ \\
\hline
\end{tabular}

Travel time was observed in Phase I in the four slots everyday with the help of moving car method by repeated car trips along the route. GPS enabled phones equipped with a GPS tracking mobile application called 'GPS Tracker Lite' was used to locate regions of low speed. Clearly perceived congestion, signalized intersection and bus dwell time delays for buses ahead of the car were individually noted for reference. In Phase II, two acquainted commuters were chartered with making the GPS logs with timestamp of arrival. 


\section{DATA ANALYSIS}

\subsection{Traffic Composition}

A quick overview at the obtained data clearly revealed a dominance of passenger cars and vans in the traffic streams across all nodes, except at night off-peak time, that was marked with a high proportion of trucks. This composition gives a qualitative idea of the type of congestion. On arterial and sub-arterial roads where abundant space is available for manoeuvres, a high proportion of two wheelers means more of the road capacity may be used as they fit into the spaces between the large vehicles and reduce queuing.

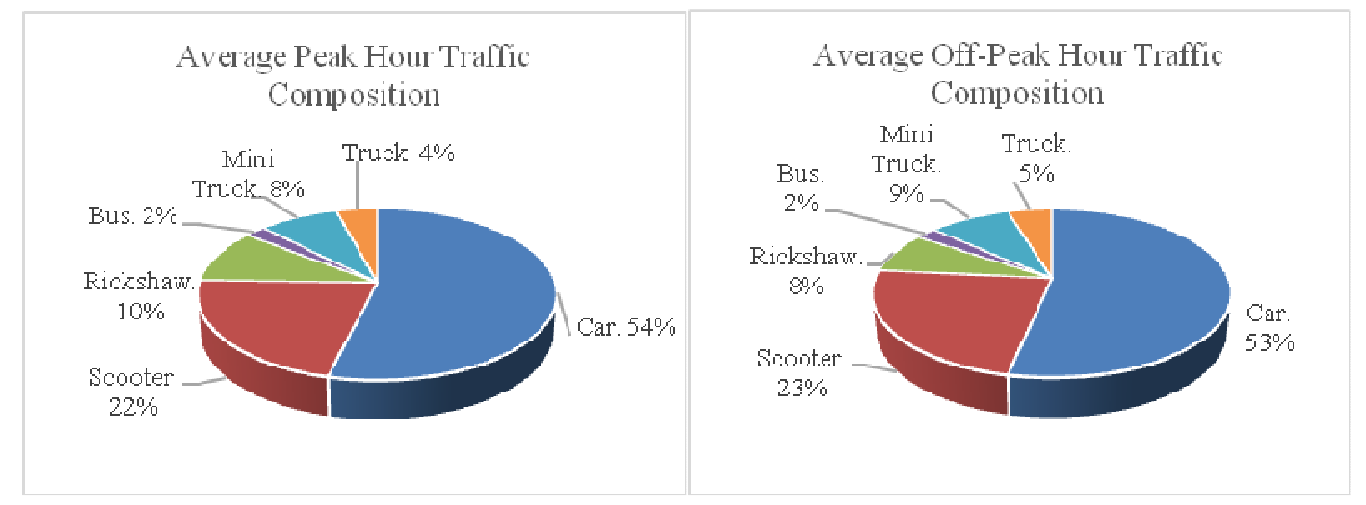

Figure 1. Average traffic composition during peak and non-peak hours

\subsection{Node and segment variables}

The manually collected were digitized and a comprehensive table containing the relevant sets was obtained after removing visible noise (absurd values). Traffic volume was converted to PCU (Passenger Car Unit), the standard unit of vehicular traffic, using the given formula as suggested by HCM 2010: -

$$
Q_{P C U}=Q * \frac{v_{c} / v_{i}}{A_{c} / A_{i}}
$$

Here $Q$ is the observed volume (in vehicles per hour), $v_{i}$ and $A_{i}$ are the average spot speed and plan area of the $\mathrm{i}^{\text {th }}$ category vehicle and $\mathrm{v}_{\mathrm{c}}$ and $\mathrm{A}_{\mathrm{c}}$ are the corresponding spot speed and plan area of cars, the standard design vehicle in traffic engineering. The table 4 was referred for the values of plan area of different vehicle categories: -

Table 4. Plan area of vehicle categories.

\begin{tabular}{|l|l|l|}
\hline Vehicle category & Plan area (sq. m.) & Area ratio $\left(\mathbf{A}_{\mathbf{c}} / \mathbf{A}_{\mathbf{i}}\right)$ \\
\hline Car & 5.36 & 1.000 \\
\hline Two-wheeler & 1.20 & 4.467 \\
\hline Three-wheeler & 4.48 & 1.196 \\
\hline LCV & 8.11 & 0.661 \\
\hline HCV (Truck) & 24.54 & 0.218 \\
\hline Bus & 24.54 & 0.218 \\
\hline
\end{tabular}


Spot speeds obtained by radar gun were averaged over the count period and then were converted to stream speed, the speed with which the average vehicle moves on that spot, equivalent to the PCU of traffic volume, using the following formula: -

$V_{s}=\frac{\sum_{i=1}^{N} v_{i} * Q_{i}}{\sum_{i=1}^{N} Q_{i}}$

Here $\mathrm{V}_{\mathrm{s}}$ is the stream speed, $\mathrm{v}_{\mathrm{i}}$ is the average spot speed and $\mathrm{Q}_{\mathrm{i}}$ the volume (vehicles per hour) of the $\mathrm{i}^{\text {th }}$ category vehicle and $\mathrm{N}$ is the number of categories $(=6)$.

The computed values of stream volume and speed at nodes were used to calculate the traffic density of the sections. Density is a traffic flow parameter that depicts the "crowdedness" of the traffic stream, an important indicator of congestion used especially in capacity based quantification of congestion: -

$$
\operatorname{Density}(K)=\frac{\operatorname{Volume}(Q)}{\text { Speed }(V)}
$$

Finally, the estimated average values of the traffic volume, speed and density across segments were computed by simply averaging the values of the origin and destination nodes.

\section{Modelling}

\subsection{Selection of variables}

The primary aim of this study being origin-destination based congestion estimation, different node variables were tested for correlation both among themselves as well as with the dependent variable - Travel Time. In the Pearson correlation matrix of the independent variables, all but five correlation coefficients came out to be between -0.5 and 0.5 (other than the definite correlation between node and averaged segment values), leading to the conclusion that most of them are really independent. Also, all but two variables, viz. 'Number of Lanes' and 'Destination Speed', were found to be reasonably related to the dependent variable, with their correlation coefficients greater than 0.5 . The values are tabulated in table 5: -

Table 5. Variables considered in the model along with their correlation coefficients with the dependent variables: Travel Time.

\begin{tabular}{|l|l|l|l|}
\hline Independent variable & Symbol & Unit & Correlation coefficient \\
\hline Roadway parameters: - & & & \\
\hline Segment length & $\mathrm{L}$ & $\mathrm{Km}$ & 0.890 \\
\hline Number of intersections & $\mathrm{N} 1$ & & 0.914 \\
\hline Number of Lanes & $\mathrm{N} 2$ & & 0.356 \\
\hline Origin - destination parameters: - & & & \\
\hline Origin volume & $\mathrm{QS}$ & $\mathrm{Pc} / \mathrm{hr}$ & 0.697 \\
\hline Origin speed & $\mathrm{VS}$ & $\mathrm{Km} / \mathrm{hr}$ & 0.600 \\
\hline Origin density & $\mathrm{KS}$ & $\mathrm{Pc} / \mathrm{km}$ & 0.549 \\
\hline Destination volume & $\mathrm{QD}$ & $\mathrm{Pc} / \mathrm{hr}$ & 0.810 \\
\hline Destination speed & $\mathrm{VD}$ & $\mathrm{Km} / \mathrm{hr}$ & 0.552 \\
\hline Destination density & $\mathrm{KD}$ & $\mathrm{Pc} / \mathrm{km}$ & 0.726 \\
\hline Segment averaged parameters: - & & & \\
\hline Segment volume & $\mathrm{Q}$ & $\mathrm{Pc} / \mathrm{hr}$ & 0.778 \\
\hline Segment speed & $\mathrm{V}$ & $\mathrm{Km} / \mathrm{hr}$ & 0.513 \\
\hline Segment density & $\mathrm{K}$ & $\mathrm{Pc} / \mathrm{km}$ & 0.660 \\
\hline
\end{tabular}




\subsection{Model Development}

With the given data, two datasets were created: one with the source-destination values, the other one with segment values averaged from the first set. Both linear and exponential multivariate models were developed for each of the two datasets with the help of IBM SPSS, a statistical analysis software application. The general form of the two types of models is: -

Linear Model: $y=\sum_{i=1}^{n} a_{i} x_{i}+c$

Exponential Model: $y=\sum_{i=1}^{n} a_{i} x_{i}^{b_{i}}+c$

Where $a_{i}$ and $b_{i}$ denote the regression coefficients and $c$ the constant term to be computed following regressing. Analysis of Variance (ANOVA) was carried out on the four models. F test was carried out on the models with a DDF (denominator degrees of freedom) of 383 on a confidence interval of $95 \%$. The results are given in the table 6 :

Table 6. Regression models.

\begin{tabular}{|l|l|l|l|l|l|}
\hline $\begin{array}{l}\text { Model } \\
\text { number }\end{array}$ & Regression type & Data set & $\begin{array}{l}\text { Adjusted R } \\
\text { value }\end{array}$ & F value & $\begin{array}{l}\text { P value } \\
(\mathbf{a t} \boldsymbol{\alpha}=\mathbf{0 . 0 5})\end{array}$ \\
\hline 1 & Linear & Origin-destination & 0.917 & 52.745 & 0.000 \\
\hline 2 & Linear & Segment & 0.896 & 37.012 & 0.002 \\
\hline 3 & Exponential & Origin- destination & 0.896 & 28.658 & 0.015 \\
\hline 4 & Exponential & Segment & 0.860 & 19.514 & 0.004 \\
\hline
\end{tabular}

The adjusted $\mathrm{R}^{2}$ value is a statistical parameter that depicts the proportion of variance in Travel Time, the dependent variable, explained by that of the independent variables themselves, and is a good indicator of the credibility of the model. The obtained P value of Model 1 following the Ftest indicates that its null hypothesis can be safely rejected and it may be concluded that the model is better than the one with only intercepts. Thus Model 1 , which has the highest $\mathrm{R}^{2}$ value and the best $\mathrm{F}$ value, was chosen as the desired model. The following formula of travel time (in seconds) was arrived at.

Model 1:

$T=-94.1+15.2 * L+135.6 * N_{1}+0.27 * Q_{s}-0.15 * Q_{d}-23.9 * V_{s}+26.0 * V_{d}-6.16 * K_{s}+4.89 * K_{d}$

The symbols denote the variables as mentioned in table 5 .

\subsection{Validation of Model}

Modelling by regression was carried out with $75 \%$ of the pre-processed data, while the remaining $25 \%$ was used for validation of the same model. This was done by finding out normalised root mean squared error (NRMSE) and mean absolute percentage error (MAPE), values that determine the predictive power of the model. They are given by the following formulae: -

$$
\begin{aligned}
& \text { NRMSE }=\frac{R M S E}{\bar{X}_{o}} \\
& \text { RMSE }=\sqrt{\frac{\sum_{i=1}^{N}\left(X_{o_{i}}-X_{p_{i}}\right)^{2}}{N}} \\
& \text { MAPE }=\frac{100}{N} \sum_{i=1}^{N}\left|\frac{X_{o_{i}}-X_{p_{i}}}{X_{o_{i}}}\right|
\end{aligned}
$$


Here $X_{o}$ is the actual value of the parameter, $X_{p}$ is its predicted value according to the model, is the mean of the observed values and $\mathrm{N}$ is the total number of observations in the validation dataset. A low value of these values is desirable; typically, a value of around $0.1(10 \%)$ of NRMSE and MAPE depict a highly accurate model. The obtained values on the validation dataset in this linear source-destination (Model 1) model are given in table 7.

The values of NRMSE and MAPE imply a substantially accurate forecasting. From the Predicted Time vs. Observed Time graph in Figure 2, it may be understood that the applied model works well for the validation dataset.

Table 7. Validation dataset parameters.

\begin{tabular}{|l|l|}
\hline Property & Value \\
\hline Number of observations, $\mathrm{n}$ & 96 \\
\hline Mean observed travel time, $\mathrm{T}_{\mathrm{o}}(\mathrm{sec})$ & 580 \\
\hline Mean predicted travel time, $\mathrm{T}_{\mathrm{p}}(\mathrm{sec})$ & 572 \\
\hline RMSE $(\mathrm{sec})$ & 41.8 \\
\hline NRMSE (\%) & 7.20 \\
\hline MAPE (\%) & 8.56 \\
\hline
\end{tabular}

\section{FREE FLOW AND CONGESTION}

The finalised model gives an estimate of the travel time in each trip on a given segment or the entire route, but it does not conclusively depict the state of congestion on the segment. As discussed earlier, congestion index is a sensible measure of congestion and uses both actual travel time as well as the ideal free flow time, the latter simply being the segment length divided by free flow speed (FFS).

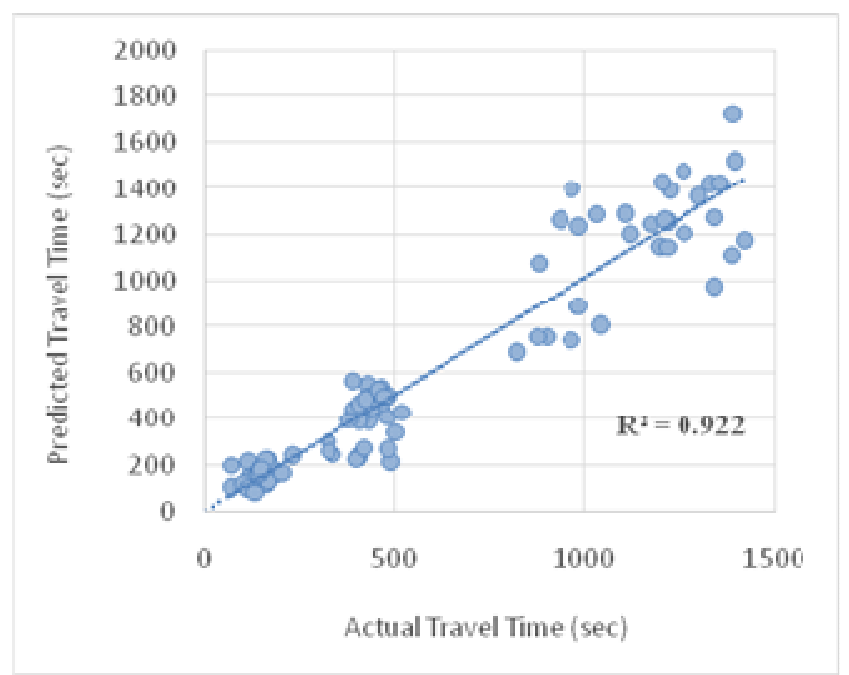

Figure 2. Predicted versus observed travel time results of validation of the travel time model

Different empirical formulae/values of FFS have been suggested by various scholars. This study relies on the hypothesis that the choice of correctness of the free flow speed is independent of the travel time that may be expected in a general non-free flow condition and does not affect the value of congestion index substantially, meaning the assumption that any of the methods for calculating FFS theorized before (see Literature Review) may be used in calculating CI may be 
held valid. For this study, Schrank and Lomax's (2005) approximate constant value of $35 \mathrm{mph}(\approx$ $55 \mathrm{kmph}$ ) for urban arterials was used.

With this information, the average value of Congestion Index (CI) in the peak hour was calculated for each segment with the help of the following formula: -

$$
C l=\frac{T-L / V_{f}}{L / V_{f}}
$$

Where $\mathrm{T}$ is the average of the travel times as computed by the model on the validation dataset, $\mathrm{L}$ is the segment length and $\mathrm{V}_{\mathrm{f}}$ is taken as $55 \mathrm{kmph}$.

The values obtained for the morning and evening peak hours (slots 1 and 3 ) congestion state in the study route is shown in table 8 and in Figure 3: -

Table 8. Congestion Index values.

\begin{tabular}{|l|l|l|}
\hline Segment number & Morning peak CI value & Evening peak CI value \\
\hline 1 & 0.62 & 0.58 \\
\hline 2 & 2.40 & 2.18 \\
\hline 3 & 3.24 & 3.25 \\
\hline 4 & 1.53 & 1.44 \\
\hline 5 & 1.15 & 1.10 \\
\hline 6 & 1.12 & 1.05 \\
\hline
\end{tabular}

The values obtained imply that segment 2 and 3 (i.e., Sarai Kale Khan to Andrews Ganj to AIIMS North) remain extremely congested especially during the evening peak time, with as high an average value of 2.71 implying that an about $81 / 2$ minutes long commute at midnight usually takes about 32 minutes during the evening.

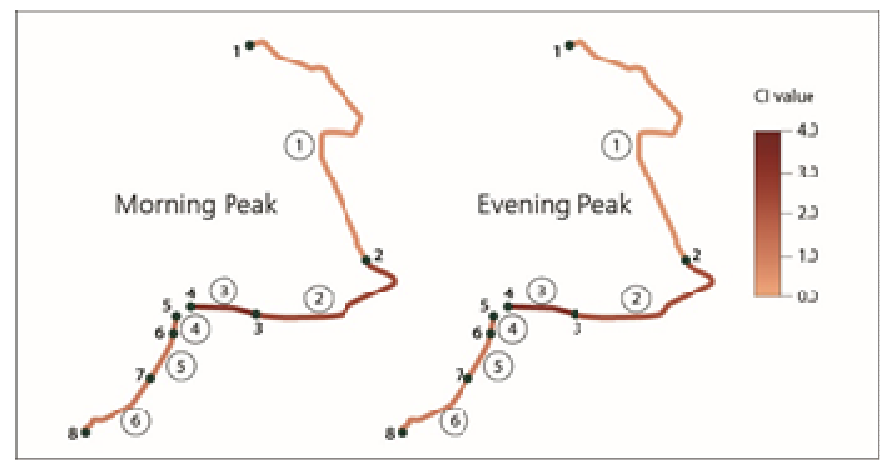

Figure 3. Segment wise route map by CI value. The darker segments indicate higher level of congestion

The obtained values of congestion index are significantly higher than typical values of cities as a whole, as suggested by TomTom, which classifies cities with CI values above 0.5 as reasonably congested. This is partly attributed to the nature of the study with specific focus on busy arterials instead of the entire road network that in general faces a lesser degree of congestion. However, with this study it is clearly established that the south-eastern portion of the Inner Ring Road of Delhi (study segments 2 and 3) becomes extraordinarily congested at peak times and thus needs to be immediately checked for congestion reduction measures. 


\section{CONCLUSIONS}

This paper proposes an idea of estimating congestion on urban roads with reduced cost of field data collection by limiting observation sites at only a few select points (nodes) in the route instead of the entire length.

A model for prediction of travel time on a given segment was prepared using multiple linear regression. With the knowledge of the roadway parameters such as length and number of minor intersections/diversion routes and by measuring the traffic parameters of the nodes (start and end) of the segments, the expected travel time can be estimated. Combined with the knowledge of free flow time for that segment, which from among other methods can also be approximated from the midnight field observation data, Congestion Index, which is an efficient, route length independent measure of congestion, can be calculated. This procedure was carried out in this study for a major road route in Delhi. Data for travel times by a bus and category wise spot speed and volume were collected, analysed and used for modelling, as well as for validation of the same model. Different combinations of situations were analysed to arrive at four efficient models, of which one with the best statistical values was chosen (adjusted R2 $=0.917$ ). The model was validated with the help of root mean squared error (RMSE) evaluation, which with a value of $7.2 \%$ was found remarkably reliable.

It can also be seen that this study, due to the virtue of scope, has some limitations that were duly noted. The use of node data to estimate travel time may help in estimation of travel time, but it falters in providing help for suggesting alternative routes because the node data for alternative routes remain the same notwithstanding anything but roadway parameters such as length and diversions. In order to make this distinction clear, more roadway parameters should be studied for influence on traffic congestion.

\section{REFERENCES}

[1] Aftabuzzaman, Md. (2007) "Measuring Traffic Congestion - A Critical Review", 30th Australasian Transport Research Forum.

[2] Anjaneyulu, M.V.L.R. \& Nagaraj, B.N. (2009) "Modelling Congestion on Urban Roads using Speedprofile data", Journal of the Indian Roads Congress, Paper no. 549.

[3] Bharati, A.K., Gopi, P., \& Sachdeva, S.N. (2014) “Evaluation of Travel Time Reliability on Urban Arterial”, International Journal of Engineering Research \& Technology (IJERT), Vol. 3, Issue 6 ISSN: 2278-0181.

[4] Bovy, P. H. L., \& Salomon, I. (2002) "Travel Behaviour: Spatial Patterns, Congestion and Modelling", Chapter 8: Congestion in Europe: Measurements, Patterns and Policies. E. Stern, I. Salomon and P.H.L. Bovy (eds.).

[5] Chakrabartty A., \& Gupta S. (2015) "Estimation of Congestion Cost in the City of Kolkata - a Case Study", Current Urban Studies 3: pp 95-104. Available from internet: <http://www.scirp.org/journal/cushttp://dx.doi.org/10.4236/cus.2015.32009>

[6] Coscia, V., Delitala, M., \& Frasca, P. (2007) "On the Mathematical Theory of Vehicular Traffic Flow II Discrete Velocity Kinetic Models”, International Journal of Non-Linear Mechanics, Elsevier, Vol. 42, Issue 3, pp. 411. 
Civil Engineering and Urban Planning: An International Journal (CiVEJ ) Vol.4, No.2, June 2017

[7] Das, A.K., Saw, K., \& Katti, B.K. (2016) "Traffic Congestion Modelling with Reference to Speed Profiles under Mixed Traffic Conditions: A Case Study of Surat Corridor", Global Research and Development Journal for Engineering । Recent Advances in Civil Engineering for Global Sustainability.

[8] Lomax, T., Turner, S., Shunk, G., Levinson, H.S., Pratt, R. H., Bay, P. N., \& Douglas, G.B. (1997) "Quantifying Congestion", Washington DC: Transportation Research Board 1 \& 2, NCHRP Report 398.

[9] Maitra, B., Sikdar, P., \& Dhingra, S. (1999) "Modelling Congestion on Urban Roads and Assessing Level of Service", Journal of Transpiration Engineering, Vol. 125, Issue 6, pp. 508-514.

[10] Pignataro, L. J., \& Cantilli, E. J. (1973) "Traffic Engineering: Theory and Practice", Prentice-Hall Inc., Englewood Cliffs, New Jersey.

[11] Rao, A. M., \& Rao, K. R. (2012) “ Measuring Urban Traffic Congestion - A Review”, Blekinge Institute of Technology European Spatial Planning and Regional Development, Vol. 2, Issue 4, pp. 286-305.

[12] Sarkar, P.K., Bose, S., \& Ghosh, P. (2007) "A Critical Appraisal of Traffic \& Transportation Sector in Delhi and Possible Solutions”, Journal of the Indian Roads Congress, Paper No. 532.

[13] Schrank, D. L., \& Lomax, T. J. (2005) "In the 2005 Urban Mobility Report", Texas Transportation Institute, 91p.

[14] Smeed, R. J. (1968) "Traffic Studies and Urban Congestion", Journal of Transport Economics and Policy, Vol. 2, pp. 33-70.

[15] Taylor, M. A. P. (1992) "Exploring the Nature of Urban Traffic Congestion: Concepts, Parameters, Theories and Models", In Proceedings of the 16th Conference of the Australian Road Research Board, Vol. 16, pp. 83-104.

[16] Weisbrod, G., Vary, D., \& Treyz, G. (2001) "Economic Implications of Congestion”, Washington, DC, Transportation Research Board, NCHRP Report 463. 